\title{
Íleo biliar, revisión del manejo quirúrgico
}

\author{
Gallstone ileus, surgical management review
}

\author{
Marcos I. Salazar-Jiménez ${ }^{1 *}$, Javier Alvarado-Durán², Mónica R. Fermín-Contreras³, \\ Fernando Rivero-Yáñez', Arianne I. Lupian-Angulo y Antonio Herrera-González ${ }^{1}$ \\ ${ }^{1}$ Servicio de Cirugía General; ${ }^{2}$ Servicio de Cirugía General, Sanatorio Durango; ${ }^{3}$ Servicio de Endoscopía. Hospital Central Norte PEMEX, Ciudad \\ de México, México
}

\section{Resumen}

Antecedentes: El íleo biliar (IB) es una causa poco frecuente de oclusión intestinal mecánica, causado por la impactación de un cálculo biliar en el tubo digestivo, siendo la localización más frecuente el íleon terminal; se debe al paso de un cálculo a través de una fístula bilioentérica. Debido a su baja incidencia, la sospecha diagnóstica y el tratamiento quirúrgico inicial adecuado son de gran importancia para la evolución clínica. Objetivo: Realizar una revisión bibliográfica sobre el manejo quirúrgico actual del IB y ejemplificarlo mediante la presentación de un caso clínico. Caso clínico: Varón de 78 años con cuadro de oclusión intestinal, con presencia de cálculo biliar en el íleon distal por tomografía. Se realiza laparotomía exploradora con enterotomía y extracción del cálculo. Cursa con adecuada evolución posquirúrgica, documentándose fístula colecistoduodenal por panendoscopia. Discusión: EI IB es una patología poco común, por lo cual existe discrepancia en cuanto al tipo de manejo quirúrgico ideal, sobre todo en si se debe o no asociar un procedimiento biliar a la enterolitotomía de urgencia. Conclusión: El IB se asocia a complicaciones secundarias al retraso diagnóstico y a una mala elección de la técnica quirúrgica inicial. Si bien el tratamiento está encaminado a resolver la obstrucción intestinal mediante enterotomía y extracción del cálculo biliar, existe controversia en cuanto al tiempo preferido para realizar la colecistectomía y la reparación de la fístula bilioentérica, siendo la cirugía en dos tiempos el procedimiento quirúrgico de elección, sobre todo en pacientes con alto riesgo de complicaciones.

PALABRAS CLAVE: Íleo biliar. Manejo quirúrgico. Fístula colecistoduodenal. Oclusión intestinal.

\begin{abstract}
Background: Gallstone ileus (Gl) represents a rare cause of mechanical intestinal occlusion, which is caused by the impaction of a gallstones at the gastrointestinal tract, being most frequently the terminal ileum; its etiology is due to the passage of a calculum through a biliary-enteric fistula. Due to its low incidence, diagnostic suspicion and adequate initial surgical treatment are essential for an adequate clinical evolution. Objective: $A$ bibliographic review on the current surgical management of GI was carried out and exemplified by the presentation a clinical case. Clinical case: 78-year-old male with bowel obstruction, upon undergoing a CT scan, a gallstone at the level of distal ileum is displayed, therefore, an exploratory laparotomy (ex lap) is performed with enterotomy and extraction of the calculus. The patient bestowed adequate postoperative clinical evolution, and the presence of a cholecystoduodenal fistula is documented by an upper endoscopy. Discussion: GI represents an uncommon pathology, however, there is discrepancy in the literature regarding the initial surgical management, especially in whether or not a biliary procedure should be associated with emergency enterolithotomy. Conclusion: Gl is associated with complications secondary to diagnostic delay and its late surgical resolution, although the initial treatment is aimed at resolving
\end{abstract}

\author{
Correspondencia: \\ *Marcos I. Salazar-Jiménez \\ Valladolid 40 interior $304 \mathrm{~A}$ \\ Col. Roma norte, Del. Cuauhtémoc \\ C.P. 06700, Ciudad de México, México \\ E-mail: marcos.salazarj@gmail.com
}

Fecha de recepción: 01-04-2018

Fecha de aceptación: 20-04-2018

DOI: 10.24875/CIRU.M18000032
Cir Cir. 2018;86:182-186

Contents available at PubMed www.cirugiaycirujanos.com 
the intestinal obstruction through enterotomy and gallstone extraction, there is controversy regarding the preferred time for cholecystectomy and repair of biliary-enteric fistula, being the two-stage surgery the surgical procedure of choice, especially in patients with a high risk of complications.

KEY WORDS: Gallstone ileum. Surgical management. Cholecystoduodenal fistula. Intestinal occlusion.

\section{Introducción}

El íleo biliar (IB) se describe como una obstrucción intestinal mecánica causada por la impactación de uno o más cálculos biliares en cualquier parte del tubo digestivo, principalmente en el intestino delgado, siendo el sitio más común el íleon distal'1. La etiología es secundaria al paso de un cálculo biliar desde los conductos biliares o la vesícula biliar hacia el tracto gastrointestinal a través de una fístula enterobiliar (siendo la causa más común la fístula colecistoduodenal), la cual se forma por la presencia de episodios recurrentes de colecistitis aguda o secundaria al síndrome de Mirizzi, en el cual existe inflamación perivesicular crónica, generando adherencias entre la vía biliar y el tracto gastrointestinal, y posterior a ello la presión ejercida por el cálculo biliar produce necrosis de la pared vesicular, erosionándola y creando la comunicación bilioentérica ${ }^{2-4}$.

El IB representa del 0.3 al $0.5 \%$ de las complicaciones de la colelitiasis, con una incidencia de $3 / 100,000$ hospitalizaciones por patología biliar, siendo más frecuente en el sexo femenino en una relación 3.5:1, con una mayor incidencia en mayores de 65 años. Representa el $0.5 \%$ de las causas de obstrucción del intestino delgado, afectando principalmente a pacientes mayores de 65 años con múltiple comorbilidad, y con una mortalidad de 5 a 10 veces mayor que el resto de las causas de obstrucción de intestino delgado ${ }^{5,6}$.

El tamaño del cálculo es significativo para desencadenar la enfermedad. Se describe que debe medir por lo menos $2 \mathrm{~cm}$ de diámetro $(2.5 \mathrm{~cm}$ en promedio) para causar obstrucción ( $90 \%$ de los casos). El sitio donde se aloje el cálculo inducirá diferentes escenarios clínicos, entre los que se describe el síndrome de Barnard, en el cual el cálculo obstruye la válvula ileocecal, manifestándose como un cuadro de oclusión intestinal clásico, en ocasiones acompañado de ictericia (menos del 15\%), caracterizado por distensión abdominal, dolor, vómito, ausencia de peristalsis, constipación u obstipación. Por otro lado se encuentra el síndrome de Karewsky, el cual se caracteriza por la presencia crónica de dolor abdominal intermitente, causado por el paso de cálculos biliares al intestino. La afección del colon es mucho más rara, asociándose a sitios de estenosis o estrechez secundaria a enfermedad inflamatoria intestinal|2,-9.

La sospecha diagnóstica es de suma importancia para un manejo oportuno. Clínicamente se describe la tríada de Mordor, que incluye diagnóstico de colelitiasis conocido, signos clínicos de colecistitis aguda y datos compatibles con obstrucción intestinal. Como parte de los estudios paraclínicos se describe en la radiografía de abdomen la tríada de Rigler, la cual consiste en la presencia de cálculos radioopacos (menos del $10 \%$ de los casos), neumobilia (signo de Gotta-Mentschler) y distensión de las asas intestinales, estableciéndose el diagnóstico ante la presencia de dos de los tres signos radiológicos mencionados. Al agregarse el cambio de posición del cálculo biliar en una segunda radiografía se forma la tétrada de Rigler, con mayor sensibilidad diagnóstica. El signo de Petren corresponde al paso del medio de contraste hacia el tracto biliar-10.

La tomografía computarizada con medio de contraste se considera el método de elección para el diagnóstico de IB, con una sensibilidad mayor del $90 \%$. Es importante descartar aquellas causas más comunes de oclusión intestinal, como lo son las adherencias, la hernia incarcerada/estrangulada, el tumor abdominal, el vólvulo, etc. ${ }^{7,8,11}$.

El tratamiento del IB está encaminado a resolver la obstrucción intestinal, previa estabilización del paciente. El tratamiento quirúrgico continúa siendo el manejo de elección en estos pacientes y se divide en dos etapas ${ }^{1,3,8}$ :

- Etapa 1: laparotomía con enterolitotomía longitudinal en el borde antimesentérico, previo ordeñamiento del cálculo en la zona proximal, realizando un cierre transversal para evitar estenosis. No se recomienda el ordeñamiento del cálculo biliar hacia el colon debido a que se asocia con lesión de la mucosa y rotura de la serosa, la cual puede pasar inadvertida ${ }^{1,2}$. Se realiza una revisión sistemática de todo el intestino en búsqueda de cálculos biliares, que pueden encontrarse en el 3-16\% de los casos, pudiendo ser extraídos por la misma enterotomía. La mayoría de los casos 
de recidiva de IB se asocian a cálculos no identificados en la laparotomía inicial. En pacientes seleccionados puede realizarse un abordaje laparoscópico con eventración del segmento intestinal afectado, lo cual representa menor morbilidad y un tiempo de recuperación más corto; sin embargo, se considera un reto técnico debido al grado de dificultad ${ }^{1,2}$.

- Etapa 2: dependiendo de los factores de riesgo del paciente, se opta por una de las siguientes opciones:

- Observación en aquellos pacientes con alto riesgo (ASA III o IV), en estado de choque, con múltiples adherencias e importante inflamación intestinal. En caso de presentar recidiva del IB (4.7-17\%) se realizará una colecistectomía laparoscópica cuando las condiciones del paciente lo permitan.

- Los pacientes con bajo riesgo (ASA I, II) pueden ser sometidos a colecistectomía y cierre de la fístula biliar en el mismo tiempo quirúrgico de la enterolitotomía con exploración de la vía biliar común si lo amerita (cirugía en un tiempo), con lo cual disminuye la recurrencia, se previenen la malabsorción y la pérdida de peso, se evitan casos de colecistitis y colangitis, y disminuye el riesgo de carcinoma de vesícula biliar ${ }^{1,2,8,12}$.

En ocasiones, el manejo endoscópico con litotricia mecánica por fragmentación puede emplearse como alternativa para aquellos pacientes que no son candidatos a manejo quirúrgico ${ }^{12,13}$.

Se trata de una patología que afecta principalmente a adultos mayores que asocian múltiple comorbilidad, presentando una mortalidad estimada del 4.5 al $25 \%$, lo que supone 5 a 10 veces mayor mortalidad en comparación con el resto de las causas de obstrucción de intestino delgado ${ }^{1,2}$.

\section{Caso clínico}

Se trata de paciente varón de 78 años de edad que ingresa proveniente del servicio de urgencias por un cuadro de 24 horas de evolución caracterizado por dolor abdominal tipo cólico, localizado en el mesogastrio, con una intensidad de 7 sobre 10 en la escala visual analógica, con irradiación difusa al resto del abdomen, el cual se asocia a intolerancia a la vía oral con presencia de vómito, en un inicio de contenido gástrico, pero que se torna de características fecaloides; refiere haber presentado una evacuación disminuida en consistencia antes de su ingreso.

Como antecedentes de importancia presenta hipertensión arterial sistémica de 16 años de diagnóstico en manejo con amlodipino ( $5 \mathrm{mg} / 24 \mathrm{~h}$ ), candesartán ( $8 \mathrm{mg} / 12 \mathrm{~h}$ ) y metoprolol $(25 \mathrm{mg} / 24 \mathrm{~h}$ ); e hipotiroidismo de 15 años de diagnóstico en manejo con levotiroxina (100 mg/24 h). Operado de apendicectomía convencional hace 6 años. Niega antecedentes traumáticos, transfusiones y toxicomanías. Refiere diagnóstico de colelitiasis desde hace 6 años por cuadro de aparente colecistitis aguda.

A la exploración presenta como hallazgos de relevancia distensión abdominal con dolor a la palpación media y profunda generalizado, de predominio en el mesogastrio, sin resistencia muscular ni datos de irritación peritoneal; peristalsis disminuida en los cuatro cuadrantes.

En sus paraclínicos presenta leucocitos $14.8 \times 10^{3} / \mathrm{ml}$, con neutrófilos $12.7 \times 10^{3} / \mathrm{ml}$, linfocitos $1.2 \times 10^{3} / \mathrm{ml}$, hemoglobina $17.3 \mathrm{~g} / \mathrm{dl}$, hematocrito $53.3 \%$, plaquetas $216 \times 10^{3} / \mathrm{ml}$, creatinina $1.22 \mathrm{mg} / \mathrm{dl}$, nitrógeno ureico $20.4 \mathrm{mg} / \mathrm{dl}$, urea $43.7 \mathrm{mg} / \mathrm{dl}$, sodio $141 \mathrm{mEq} / \mathrm{l}$, potasio $4.4 \mathrm{mEq} / \mathrm{l}$ y cloro $99 \mathrm{mEq} / \mathrm{l}$.

Se coloca una sonda nasogástrica y se documenta la presencia de gasto de tipo fecaloide de aproximadamente $1500 \mathrm{cc}$ al momento de su colocación. Se decide realizar tomografía abdominal contrastada, en la cual se reporta una imagen heterogénea a nivel del íleon, con dimensiones de $2.5 \times 2.4 \times 4.4 \mathrm{~cm}$, la cual condiciona una dilatación de las asas del intestino delgado de hasta $4.3 \mathrm{~cm}$ a nivel del yeyuno, en probable relación con IB, y presencia de dos imágenes similares de menor tamaño a nivel del yeyuno que no condicionan datos de obstrucción (Fig. 1).

Es sometido a laparotomía exploradora, en la cual se observa la presencia de un plastrón de aspecto inflamatorio en el ángulo hepático con adherencias firmes del colon transverso al duodeno, el epiplón y la cápsula de Gleason, sin lograr visualizar la vesícula biliar ni la vía biliar extrahepática. Se encuentra un cálculo biliar de aproximadamente $2.5 \times 2.5 \times 4 \mathrm{~cm}$ de diámetro localizado a $240 \mathrm{~cm}$ del ligamento de Treitz, el cual condiciona dilatación próxima de asas del intestino delgado, sin evidencia de necrosis de la pared ni disrupción de la serosa intestinal. Se realiza enterotomía longitudinal en el borde antimesentérico y se extrae el cálculo previo ordeñamiento proximal a $60 \mathrm{~cm}$ de la válvula ileocecal (Fig. 2 A y B).

El paciente cursa con adecuada evolución clínica, tolerando la vía oral 48 horas después de la intervención 

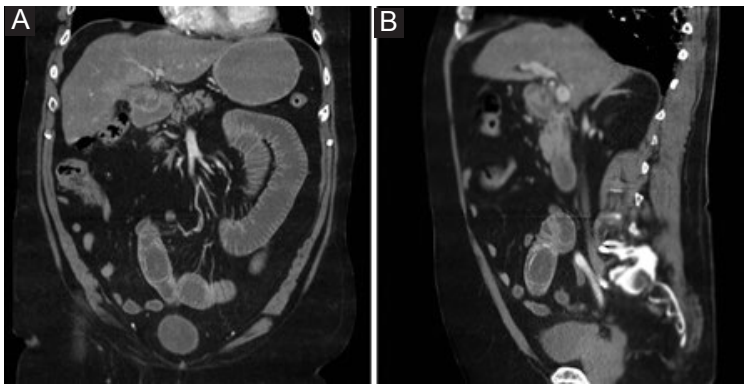

Figura 1. Tomografía abdominal en la cual se observa una imagen de $2.5 \times 2.4 \times 4.4 \mathrm{~cm}$ a nivel del yeyuno. A: corte coronal. B: corte sagital.

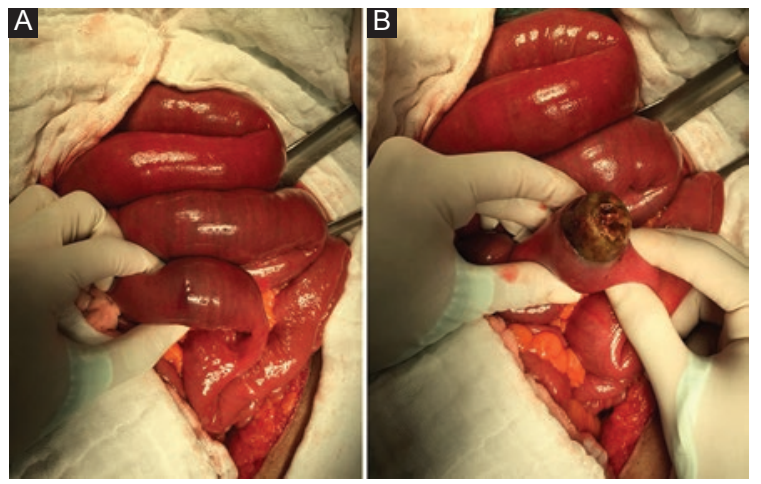

Figura 2. A: cálculo en el yeyuno con dilatación de asas a nivel proximal. B: enterolitotomía longitudinal de yeyuno con extracción del cálculo biliar.

quirúrgica. Se realiza panendoscopia y se observa la presencia de una cicatriz en contracción y orificio de fístula de aproximadamente $6 \mathrm{~mm}$ de diámetro, de bordes bien definidos, ligeramente elevados, con edema e hipertermia, con patrón vascular normal, a nivel del bulbo duodenal (Fig. 3) en relación con probable fístula colecistoduodenal.

\section{Discusión}

El IB es una de las causas poco comunes de oclusión intestinal mecánica ${ }^{1,2}$. La sospecha clínica debe estar presente ante aquellos pacientes que presentan un cuadro de dolor abdominal compatible con colecistitis aguda y datos de oclusión intestinal alta, debido a que el sitio más común de impactación del cálculo biliar es el íleon distal; solo los pacientes que presentan alteraciones en la anatomía normal del intestino grueso (enfermedad inflamatoria intestinal, neoplasias, etc.) se presentan con datos de oclusión intestinal baja por impactación del cálculo en el sitio de estrechez $z^{1,2,4}$.

El manejo inicial de los pacientes con IB es siempre quirúrgico previa estabilización (adecuada hidratación, analgesia, corrección hidroelectrolítica, etc.), y

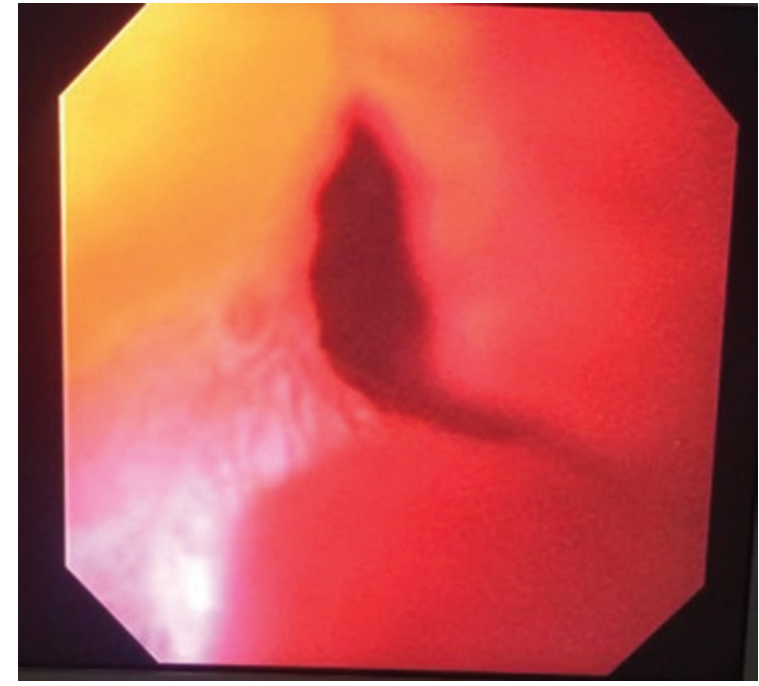

Figura 3. Endoscopia de control con presencia de fístula colecistoduodenal.

está encaminado a resolver la obstrucción y prevenir complicaciones como isquemia de la pared intestinal, perforación, peritonitis e incluso la muerte. La enterotomía en el borde antimesentérico con extracción del cálculo biliar y cierre transversal es el manejo estandarizado de urgencia; la colecistectomía con resolución de la fístula en el mismo acto quirúrgico se reserva para aquellos pacientes jóvenes y clínicamente estables, cuando sus condiciones lo permitan, siendo la cirugía en dos tiempos con colecistectomía de intervalo la más recomendada en la mayoría de los $\operatorname{casos}^{3,5}$.

\section{Conclusiones}

Debido a su baja incidencia, no existe un consenso que paute el manejo a seguir en los pacientes con diagnóstico de IB. Si bien se sabe que el tratamiento estándar es la resolución quirúrgica, hay diversas opiniones en cuanto al tipo de cirugía a realizar. Reisner y Cohen ${ }^{14}$ apoyan el manejo quirúrgico en dos tiempos con enterolitotomía más extracción del cálculo de urgencia, con lo cual se resuelve la obstrucción intestinal, asociado a una colecistectomía de intervalo con reparación de la fístula colecistoentérica en 4-6 semanas en pacientes con riesgo de recurrencia, la cual se asocia a una menor morbilidad. Por otro lado, Rodríguez-Sanjuán. et al. ${ }^{15}$ recomiendan la cirugía en un solo tiempo, en la cual se incluye una técnica biliar (colecistectomía o colecistostomía), con cierre de la fístula asociado a la enterolitotomía, argumentando una disminución en el riesgo de recurrencia y de carcinoma de vesícula biliar, con una 


\section{Diagnóstico de IB}

\section{Enterotomía con extracción del cálculo biliar* \\ (Laparotomía exploradora o laparoscópica)}

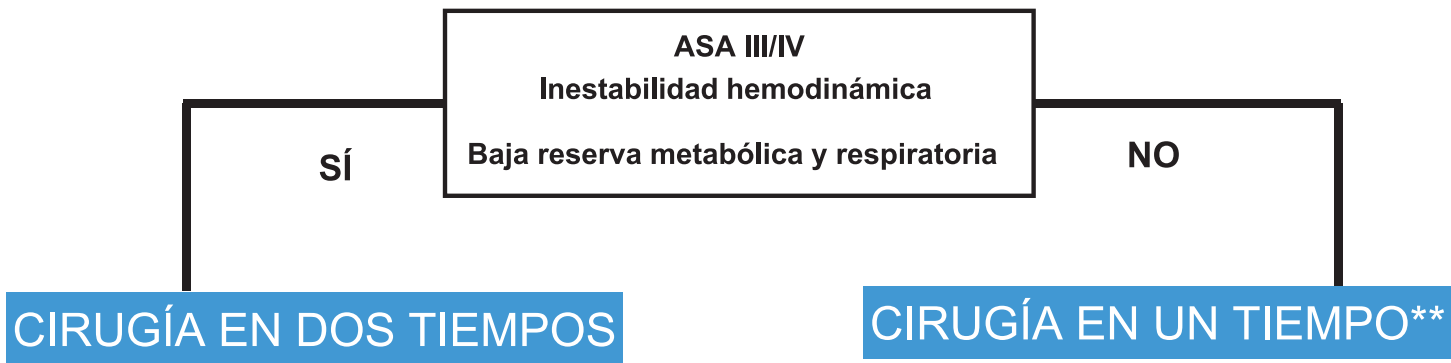

Revisión sistemática del resto del intestino Y extracción de cálculos por ordenamiento

\section{COLECISTECTOMÍA COLECISTOSTOMÍA}

\section{COLECISTECTOMÍA DE INTERVALO*** REPARACIÓN DE LA FÍSTULA}

Figura 4. Algoritmo terapéutico del íleo biliar.

mortalidad similar a la de la enterolitotomía sola, sobre todo en pacientes con estabilidad clínica y bajo riesgo quirúrgico. El manejo endoscópico se reserva para aquellos pacientes con alto riesgo quirúrgico.

De acuerdo con la revisión de la bibliografía ${ }^{1,8}$, nosotros proponemos el algoritmo terapéutico que se muestra en la figura 4.

\section{Conflicto de intereses}

Los autores manifiestan no tener ningún tipo de conflicto de intereses.

\section{Bibliografía}

1. Halabi WJ, Kang CY, Ketana N, Lafaro KJ, Nguyen VQ, Stamos MJ, et al. Surgery for gallstone ileus: a nationwide comparison of trends and outcomes. Ann Surg. 2014;259:329-35.

2. Van Hillo M, van der Vliet JA, Wiggers T, Obertop H, Terpstra OT, Greep JM. Gallstone obstruction of the intestine: an analysis of ten patients and a review of the literature. Surgery. 1987;101:273-6.

3. Beltran MA, Csendes A, Cruces KS. The relationship of Mirizzi syndrome and cholecystoenteric fistula: validation of a modified classification. World J Surg. 2008;32:2237.
4. Martín Pérez J, Delgado Plasencia L, Bravo Gutiérrez A, Burillo-Putze G, Martínez-Riera A, Alarcó-Hernández A, et al. [Gallstone ileus as a cause of acute abdomen. Importance of early diagnosis for surgical treatment.] Cir Esp. 2013:91:485-9.

5. Alexiou K, loannidis A, Sikalias N, Konstantinidou E, Fotopoulos A, Karanikas I, et al. Gallstone ileus: a case report and our clinic's experience. Surgical Science. 2014:5:10-4.

6. Ayantunde AA, Agrawal A. Gallstone ileus: diagnosis and management. World J Surg. 2007;31:1292.

7. Beuran M, Ivanov I, Venter MD. Gallstone ileus-clinical and therapeutic aspects. J Med Life. 2010;3:365-71.

8. Keaveny AP, Afdhal NH, Bowers S. Gallstone ileus. UpToDate. Disponible en: https://www.uptodate.com/contents/gallstone-ileus.

9. Rodríguez-Hermosa JI, Codina-Cazador A, Gironès-Vilà J, Roig García J, Figa Francesch M, Acero Fernández D. Íleo biliar: resultados del análisis de una serie de 40 casos. Gastroenterol Hepatol. 2001;24:489-94.

10. Noriega-Maldonado O, Bernal-Mendoza LM, Rivera-Nava JC, Guevara-Torres L. Íleo biliar. Cir Ciruj. 2005;73:443-8.

11. Doko M, Zovak M, Kopljar M, Glavan E, Ljubicic N, Hochstädter H. Comparison of surgical treatments of gallstone ileus: preliminary report. World J Surg. 2003;27:400-4.

12. Seal EC, Creagh MF, Finch PJ. Gallstone ileus: a new role for abdominal computed tomography. Postgrad Med J. 1995;71:313.

13. Nuño-Guzmán CM, Arróniz-Jáuregui J, Moreno-Pérez PA, Chávez-Solís EA, Esparza-Arias N, Hernández-González Cl. Gallstone ileus: one-stage surgery in a patient with intermittent obstruction. World J Gastrointest Surg. 2010;2:172-6.

14. Reisner RM, Cohen JR. Gallstone ileus: a review of 1001 reported cases. Am Surg. 1994;60:441-6.

15. Rodríguez-Sanjuán JC, Casado F, Fernández MJ, Morales DJ, Naranjo A. Cholecystectomy and fistula closure versus enterolithotomy alone in gallstone ileus. Br J Surg. 1997;84:634-7. 